\title{
Ophiopogonin B induces apoptosis, mitotic catastrophe and autophagy in A549 cells
}

\author{
MEIJUAN CHEN $^{1,2}$, YUANYUAN GUO ${ }^{1,2}$, RUOLIN ZHAO $^{1}$, \\ XIAOXIA WANG ${ }^{2}$, MIAO JIANG ${ }^{1}$, HAIAN FU ${ }^{3,4}$ and $\mathrm{XU}_{\text {ZHANG }}^{2}$
}

\begin{abstract}
${ }^{1}$ The Pre-Clinical Medicine College and ${ }^{2}$ Jiangsu Collaborative Innovation Center of Traditional Chinese Medicine (TCM) Prevention and Treatment of Tumor, Nanjing University of Chinese Medicine, Nanjing 210023, P.R. China; Departments of

${ }^{3}$ Pharmacology and ${ }^{4}$ Hematology and Medical Oncology, Emory University School of Medicine, Atlanta, GA 30322, USA
\end{abstract}

Received February 27, 2016; Accepted April 9, 2016

DOI: $10.3892 /$ ijo.2016.3514

\begin{abstract}
Ophiopogonin B (OP-B), a saponin compound isolated from Radix Ophiopogon japonicus, was verified to inhibit cell proliferation in numerous non-small cell lung cancer (NSCLC) cells in our previous study. However, the precise mechanisms of action have remained unclear. In the present study, we mainly investigated the effects of OP-B on adenocarcinoma A549 cells to further elaborate the underlying mechanisms of OP-B in different NSCLC cell lines. Detection by high content screening (HCS) and TUNEL assay verified that OP-B induced apoptosis in this cell line, while detection of Caspase-3, Bcl-2 and Bax showed that OP-B induced cell death was caspase and mitochondrial independent. Further experiments showed that OP-B induced cell cycle arrest in the $\mathrm{S}$ and G2/M phases by inhibiting the expression of Myt1 and phosphorylation of Histone H3 (Ser10), which resulted in mitotic catastrophe in the cells. Transmission electron microscopy (TEM) observation of cell micro-morphology combined with detection of Atgs by western blot analysis showed that OP-B induced autophagy in this cell line. Autophagy inhibition by the lysosome inhibitor CQ or Beclin1-siRNA knockdown both attenuated cell viability, demonstrated that autophagy also being the vital reason resulted in cell death. More importantly, the xenograft model using A549 cells provided further evidence of the inhibition of OP-B on tumor proliferation. Immunohistochemistry detection of LC3 and TUNEL assay both verified that high dose of OP-B $(75 \mathrm{mg} / \mathrm{kg})$ induced autophagy and apoptosis in vivo, and western blot detection
\end{abstract}

Correspondence to: Dr Haian Fu, Department of Pharmacology, Emory University School of Medicine, Atlanta, GA 30322, USA

E-mail: hfu@emory.edu

Dr Xu Zhang, Jiangsu Collaborative Innovation Center of Traditional Chinese Medicine (TCM) Prevention and Treatment of Tumor, Nanjing University of Chinese Medicine, Nanjing 210023, P.R. China E-mail: zhangxu@njutcm.edu.cn

Key words: ophiopogonin B, apoptosis, mitotic catastrophe, autophagy, cell cycle, histone $\mathrm{H} 3$ of p-Histone H3 (Ser10), Survivin and XIAP further indicated the molecular mechanism of OP-B in vivo. As our findings revealed, multiple types of cell death overlapped in OP-B treated A549 cells, it displayed multitarget characteristics of the compounds extracted from the Chinese herbal, which may be used as candidate anticancer medicine in clinic.

\section{Introduction}

Lung cancer is one of the leading causes of cancer-related deaths in the world. Non-small cell lung cancer (NSCLC) accounts for nearly $85 \%$ in all lung cancer cases, with adenocarcinoma, squamous cell carcinoma and large cell carcinoma being the main histological subtypes (1). Current clinical treatments such as surgery, radiotherapy and chemotherapy are still insufficient for NSCLC-treatment $(2,3)$ and new therapeutics need to be developed.

Traditional Chinese Medicine is efficient in improving clinical symptoms, regulating tumor growth and preventing the recurrence of lung cancer in patients. In our previous study, we found that the "Ke Jinyan Priscription", given by the State Medical Master Zhou Zhongying, inhibited the proliferation of NCI-H157 or A549 transplanted tumors, respectively, in nude mice, and the physical status of the animals was significantly improved (4). Some ingredients extracted from the formula showed significant inhibitory effects on NSCLC cell lines under screening on the high-throughput platform $(5,6)$. In particular, one of the derived compounds, ophiopogonin B (OP-B), showed especially potent inhibition of a series of NSCLC cell lines, and in $\mathrm{H} 157$ and $\mathrm{H} 460$ cell lines it mainly induced autophagic cell death which was caspase-independent (7).

Besides autophagy, the caspase-independent programmed cell death (PCD) includes paraptosis, mitotic catastrophe, and the descriptive model of apoptosis-like and necrosis-like PCD. Once caspase-mediated routes fail in vivo, caspase-independent cell death pathways are important for cleaning unwanted or harmful cells, which can also be triggered by cytotoxic agents or other death stimuli (8). As potentially new cancer therapies could be developed, the growing knowledge of the caspase-independent cell death pathways is very important for oncology research (8). 
To establish whether OP-B triggered different types of caspase-independent cell death in NSCLC cell lines, herein, we tested its effects on human lung adenocarcinoma A549 cells. The results revealed that OP-B induced mitotic catastrophe in A549 cells in vitro, and the mechanism may due to the inhibition of Myt1 and p-Histone H3 (Ser10) and the specific regulation of the cell cycle, combined with induction of autophagy, it resulted in caspase-independent apoptosis in this cell line. Moreover, OP-B inhibited expression of XIAP, survivin and the phosphorylation of Histone H3 (Ser10), thereby induced autophagy and apoptosis in vivo. Compared with its effects on H157 and H460 cells, the investigation demonstrated that OP-B may be more efficient in treating A549 cells.

\section{Materials and methods}

Reagents. Ophiopogonin B (OP-B) (MW: 722.9, HPLC $\geq 98 \%$ ) was purchased from Nanjing Zelang Medical Technology Co., Ltd. (Nanjing, China). The chemicals used were staurosporine, chloroquine (CQ), propidium iodide (PI), (3-(4,5-dimethylthiazol-2-yl)-2,5 diphenyltetrazolium bromide (MTT), DAPI and Hoechst 33258 (Sigma-Aldrich, St. Louis, MO, USA). The Alexa Fluor 488 Annexin V/Dead Cell apoptosis kit (Invitrogen, Waltham, MA, USA) and the In Situ Cell Death Detection kit (Roche, Indianapolis, IN, USA) were also obtained commercially.

Cell culture and transient transfection. The human NSCLC cell line (A549) was obtained from the Institute of Biochemistry and Cell Biology (Shanghai, China). The A549 cells were cultured in Dulbecco's modified Eagle's medium (DMEM) and F12 medium (HyClone Laboratories, Logan, UT, USA), respectively, supplemented with $10 \%$ fetal bovine serum (FBS; Gibco, Waltham, MA, USA), $100 \mathrm{U} / \mathrm{ml}$ penicillin-streptomycin mixed antibiotics at $37^{\circ} \mathrm{C}$ in a humidified $5 \% \mathrm{CO}_{2}$ incubator.

For transient transfection, A549 cells were seeded in 6-cm culture dishes at a density of $10^{6}$ cells/dish and then transfected with rat Beclin1 siRNAs (C-300506-03-0005; Dharmacon, Lafayette, CO, USA) using Lipofectamine 2000 reagent (13778-075; Invitrogen) according to the manufacturer's protocol.

Transmission electron microscopy (TEM). After being exposed to $10 \mu \mathrm{M}$ of OP-B for $48 \mathrm{~h}$, the cells were trypsinized, washed with phosphate-buffered saline (PBS) and fixed in $2.5 \%$ glutaraldehyde in $0.1 \mathrm{M}$ phosphate buffer $(\mathrm{pH} 7.2)$ overnight at $4^{\circ} \mathrm{C}$. The next day, cells were washed three times with $0.1 \mathrm{~mol} / 1$ phosphate buffer. Thereafter, the cells were fixed in $1 \%$ aqueous osmium, dehydrated with increasing concentrations of ethanol $(30,50,70,80,90$ and 100\%) and embedded in araldite. The ultrathin sections were prepared with a microtome (Leica Microsystems, Wetzlar, Germany) and mounted on copper grids. The samples were stained with $2 \%$ aqueous uranyl acetate and lead citrate and observed by TEM (JEOL, Ltd., Tokyo, Japan).

High-content screening (HCS). Apoptosis and dead cells induced by OP-B were assayed using the KineticScan Reader
(Thermo Fisher Scientific, Waltham, MA, USA). The principle of the assay is that cells are labeled with a cocktail of fluorescent dyes (including Hoechst 33258 and Alexa Fluor 488 Annexin V) that indicate the cellular properties of interest, including nuclear structure, cell membrane permeability, as well as early and late stages of apoptosis. All procedures were performed according to the manufacturer's instructions. The cells were plated at a density of $8 \times 10^{3}$ cells/ well in each well of a 96-well plate. After culturing for $24 \mathrm{~h}$, cells were incubated with $10 \mu \mathrm{M}$ OP-B for another $24 \mathrm{~h}$. Thirty minutes before the completion of incubation, a cocktail of fluorescent dyes was added to each well. The cells were then fixed with pre-warmed fixation solution and washed twice with PBS. Plates were then sealed and processed on an HCS Reader to acquire images. Images were analyzed with HCS software, and fluorescence intensities of the Hoechst 33258 and Annexin V/PI dyes were calculated. As for DAPI staining, the cells were cultured and treated and detected by HCS as above.

Western blot analysis. After treatment with different concentrations of OP-B, the cells were lysed in RIPA buffer containing $50 \mathrm{mmol} / \mathrm{l}$ Tris- $\mathrm{HCl}(\mathrm{pH} 8.0), 150 \mathrm{mmol} / \mathrm{l} \mathrm{NaCl}, 1 \%$ Nonidet-P40, $1 \%$ sodium deoxycholate, $0.1 \%$ SDS, $0.1 \mathrm{mmol} / 1$ DTT, $0.05 \mathrm{mmol} / \mathrm{l} \mathrm{PMSF}, 0.002 \mathrm{mg} / \mathrm{ml}$ aprotinin, $0.002 \mathrm{mg} / \mathrm{ml}$ leupeptin and $1 \mathrm{mmol} / 1 \mathrm{NaVO}_{3}$. The protein concentrations of supernatants were determined by the BCA protein assay. Equal amounts of protein were loaded and separated by 10 or $12 \%$ SDS-PAGE and then transferred onto polyvinylidene difluoride membranes. The membranes were incubated overnight with appropriate primary antibodies against LC3 I/II, Beclin-1, Atg3, Atg-5/12, p-Histone H3 (Ser10), caspase-3, Bcl-2, Bax, cyclin D1, cyclin D3, CDK4, CDK6, cyclin A2, CDK2, cyclin B1, Myt1, p-cdc2, p21, p27, survivin, XIAP or $\beta$-actin overnight at $4^{\circ} \mathrm{C}$, and then with HRP-conjugated secondary antibodies (anti-rabbit or mouse immunoglobulin $\mathrm{G}$ ) for an additional hour at room temperature. Immunoreactivity was detected by enhanced chemiluminescence (ECL; Bio-Rad Laboratories, Hercules, CA, USA). $\beta$-actin was used as a loading control. Immunoblot experiments were performed at least three times. Image acquisition was performed using Image $\mathrm{Lab}^{\mathrm{TM}}$ software.

Immunohistochemistry assay. The immunohistochemistry for LC3 localization in the tumor was performed as previously described (9).

Terminal deoxynucleotidyltransferase-mediated dUTP nick end labeling (TUNEL) assay. TUNEL staining was performed by using the 'In Situ Cell Death Detection kit' from Roche following the manufacturer's instructions.

Cell cycle analysis and apoptosis detection. After cells were treated with or without OP-B for $24 \mathrm{~h}$, they were harvested by centrifugation, washed with ice-cold PBS and fixed in ice-cold $70 \%$ ethanol overnight. The cells were then treated with $40 \mu \mathrm{g} / \mathrm{ml} \mathrm{RNase}$ at $37^{\circ} \mathrm{C}$ and stained with $40 \mu \mathrm{g} / \mathrm{ml}$ PI for $30 \mathrm{~min}$. The percentage of cells in each phase (sub G1, G0/G1, $\mathrm{S}$ and $\mathrm{G} 2 / \mathrm{M}$ ) was calculated (Becton-Dickinson, Franklin Lakes, NJ, USA). 
MTT assay. Cell viability was assessed by the MTT reduction assay as described by Mosmann (10). Viability of non-treated cells was taken as $100 \%$ and $\mathrm{IC}_{50}$ values were determined from three independent experiments.

Nude mouse xenografts. Five-week-old athymic BALB/c mice were maintained under specific pathogen-free conditions and manipulated according to protocols approved by the Shanghai Medical Experimental Animal Care Commission. Exponentially growing A549 cells ( $2 \times 10^{6}$ in $0.2 \mathrm{ml}$ medium) were inoculated subcutaneously into the flank of the mice. After 7 days, tumor-bearing mice were randomly divided into three groups, which were treated with OP-B $(15$ or $75 \mathrm{mg} / \mathrm{kg}$ p.o. daily; $\mathrm{n}=6$ ) or corn oil (control, $100 \mu \mathrm{l}$, p.o. daily; $\mathrm{n}=6$ ) for 21 consecutive days. Tumor growth was determined by measuring the size of the tumors every 2 days. The mice were euthanized at day 21 , and the tumors were isolated, photographed and embedded in paraffin or frozen at $-80^{\circ} \mathrm{C}$ for subsequent western blot detection of p-Histone $\mathrm{H} 3$ (Ser10), survivin and XIAP, immunohistochemical analysis of LC3 expression as well as for the TUNEL assay.

Statistical analysis. Unless otherwise stated, data are expressed as the mean \pm standard deviation (SD) and analyzed by the Student's t-test. A P-value $<0.05$ was considered statistically significant.

\section{Results}

$O P-B$ treatment induces caspase-independent apoptosis in A549 cells. Cocktail-staining by Annexin V/PI/Hoechst 33258 combined with fluorescence density analysis by HCS KineticScan Reader showed that double-positive Annexin V/PI labeling significantly occurred in A549 cells treated with 10 or $20 \mu \mathrm{M}$ of OP-B (Fig. 1A and B).

TUNEL staining assay is another effective method for marking clipped nucleic acids and nucleus-morphology observation of apoptotic cells. Compared with staurosporine (STS), a positive inducer of apoptosis which significantly induced cell chromatin condensation and TUNEL-detectable fragmentation, $10 \mu \mathrm{M}$ of OP-B treatment resulted in DNA-fragmentation moderately in A549 cells (Fig. 1D).

Further detection of apoptosis-related protein showed that OP-B-treatment did not induce activation of caspase- 3 or change the levels of Bcl-2 and Bax (Fig. 1E).

Taken together, OP-B induced DNA-fragmentation and cell death in A549 cells caspase-independently.

$O P-B$ causes cell cycle arrest and mitotic catastrophe in A549 cells. Next, flow cytometry (FCM) was used to analyze the effect of OP-B on cell cycle distribution in A549 cells. As shown in Fig. 2A, after treatment with 5,10 or $20 \mu \mathrm{M}$ of OP-B, the number of cells in the sub G1 phase increased to $6.74 \pm 0.11$, $11.30 \pm 0.88$ or $19.41 \pm 0.59 \%$, respectively, which in the vehicletreated group was only $1.07 \pm 1.30 \%$, the results were in accordance with that detected by HCS. Meanwhile, the cells in the $\mathrm{S}$ phase accumulated from $7.95 \pm 3.01$ (vehicle treated) to $14.92 \pm 1.41$ (under $10 \mu \mathrm{M}$ of OP-B) or $32.89 \pm 0.94 \%$ (under $20 \mu \mathrm{M}$ of OP-B), and the cells in the $\mathrm{G} 2 / \mathrm{M}$ phase increased from $4.49 \pm 1.18$ (vehicle treated) to $37.22 \pm 2.23$ or $28.02 \pm 0.13 \%$ under 10 or $20 \mu \mathrm{M}$ of OP-B treatment, respectively. The results suggested that OP-B induced cell death and cell cycle arrested in $\mathrm{S}$ and $\mathrm{G} 2 / \mathrm{M}$ phase.

The regulation of cell cycle transition is known to be governed by the concerted action of cyclin-dependent kinases (CDKs) and their regulatory cyclin subunits $(11,12)$. Among them, cyclin A, cyclin E and CDK2 regulate normal S-phase progression, while cyclin B1 is involved in G2-phase progression. During progression into mitosis, the critical regulatory step is dephosphorylation of cdc2 at Tyr15 and Thr14, while phosphorylation of cdc 2 by the protein kinases Weel and Myt1 results in inhibition of cdc2 (13). Moreover, phosphorylation of Histone H3 (Ser10) can drive mitotic chromosomal condensation during M phase entry (14).

After treated with 0,5 and $10 \mu \mathrm{M}$ of OP-B, the correlated proteins in the cells was detected by western blot assay. The results showed that OP-B did not affect the expression of cyclin D, CDK4, CDK6, cyclin A2, CDK2 and cyclin B1, while significantly inhibited the expression of Myt1 and the phosphorylation of cdc2 (Tyr15) and Histone H3 (Ser10). Whereas, detection of CDK inhibitors $\mathrm{p} 21^{\text {Waf } 1 / \text { Cip1 }}$ and $\mathrm{p} 27^{\mathrm{Kip} 1}$ showed that OP-B markedly increased the level of $\mathrm{p} 27^{\mathrm{Kip} 1}$ (Fig. 2B).

Cell cycle arrest in $\mathrm{G} 2 / \mathrm{M}$ phase was apt to induce mitotic catastrophe which was characterized as large cells with multiple micronuclei and decondensed charomatin. After 24-h treatment with $10 \mu \mathrm{M}$ of OP-B, A549 cells were stained with DAPI and detected by HCS, the results showed that OP-B induced formation of micronuclei in the nucleus (Fig. 2C).

Thus, we speculated that inhibition of OP-B on Myt1 and p-Histone H3 (Ser10) and enhancement of p27 ${ }^{\mathrm{Kip} 1}$ induced cell cycle arrest in $\mathrm{S}$ and $\mathrm{G} 2 / \mathrm{M}$ phase and resulted in mitotic catastrophe.

Autophagy is involved in cell death induced by OP-B in A549 cells. We have reported that OP-B induced autophagy in H157 and H460 cells (7). To investigate whether this type of cell death also occurs in OP-B treated A549 cells, we detected autophagy in this cell line. First, the micro-morphological change of OP-B treated A549 cells was observed by TEM. The results showed that OP-B treatment also resulted in numerous vacuoles in the cytoplasm (Fig. 3A). Then, detection of microtubule-associated protein 1 light chain 3 (LC3), the specific marker of autophagy showed that 12 or $24 \mathrm{~h}$ of treatment with OP-B significantly increased the transition of LC3-I to LC3-II (Fig. 3B). Atg proteins participate in the processes of autophagosome-formation (15). Synchronism detection showed that OP-B also upregulated the expression of Atg3, Atg5-Atg12 in the cells (Fig. 1B). Taken together, the results verified that OP-B also induced autophagy in A549 cells.

As is known, at the last phage of autophagy, lysosome fuses with autophagosome, then formats autophagolysosome. Chloroquine (CQ) is the inhibitor of lysosome which inhibits lysosomal degradation (16). In order to determine the role of autophagy in OP-B-induced cell death in A549 cells, the effect of CQ on OP-B-induced cell viability was initially evaluated. As shown in Fig. 3C, the decreased cell viability that resulted from $24 \mathrm{~h}$ of exposure to OP-B was effectively rescued by pretreatment with $C Q$, suggesting 
A

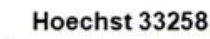

Annexin V

PI Merge

0
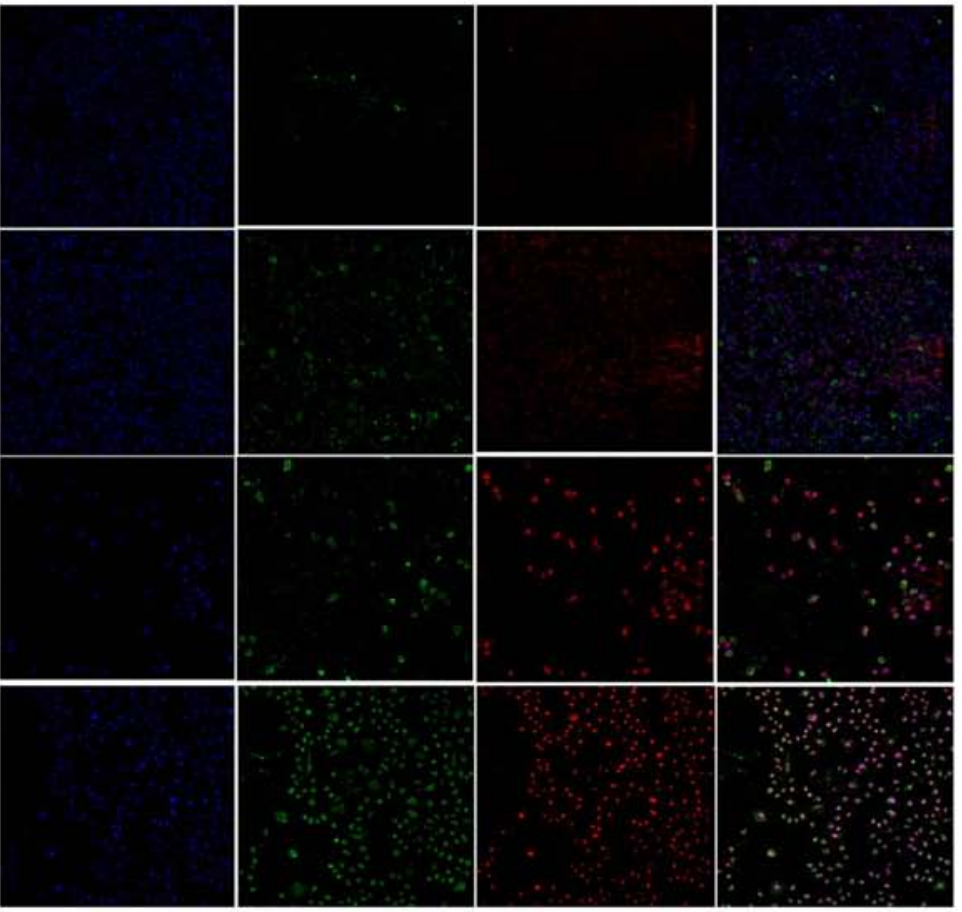

B

D

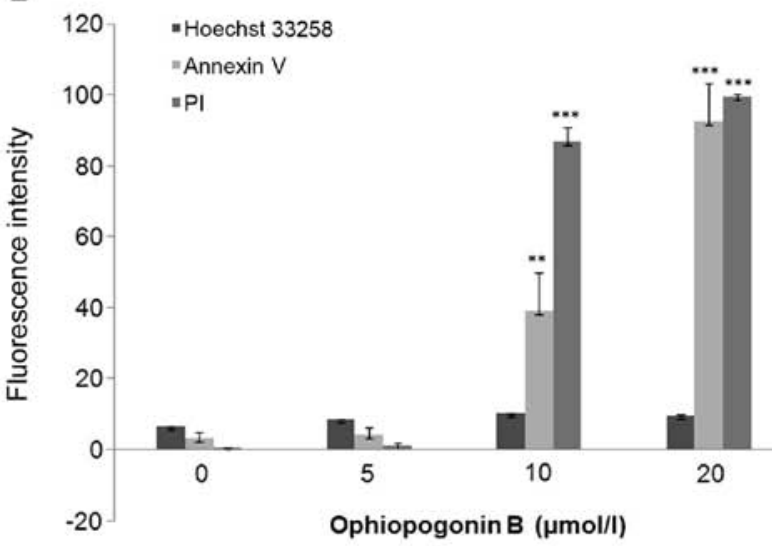

Ophiopogonin B ( $\mu$ mol/l)

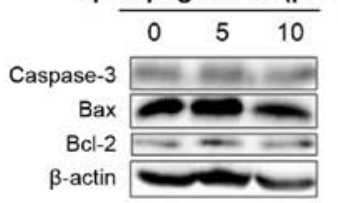

C

TUNEL

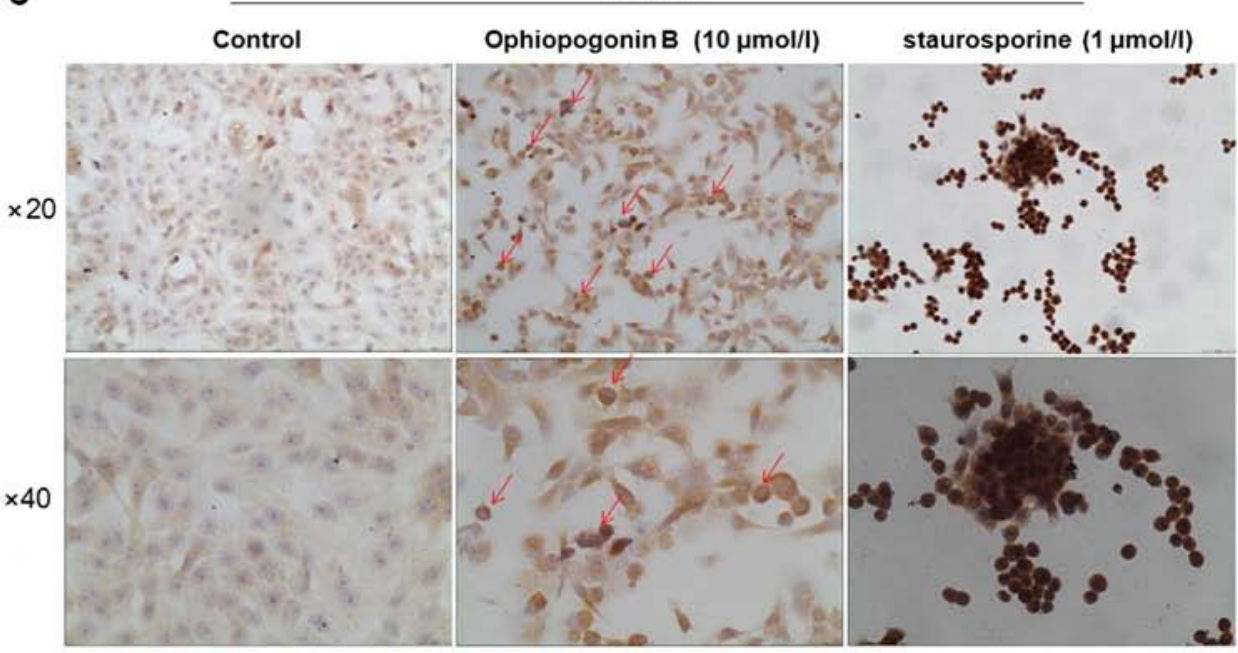

Figure 1. OP-B induces caspase-independent apoptosis in A549 cells. Cells were treated with indicated concentrations of OP-B for 24 h. (A) Morphologic changes were captured using an HCS KineticScan Reader (200) with Hoechst 33258 and Annexin V/PI staining. (B) The fluorescent intensity was quantified and analyzed by the HCS KineticScan software in three independent experiments. Error bars, SD. ${ }^{*} \mathrm{P}<0.05 ;{ }^{* * *} \mathrm{P}<0.01$; ${ }^{* * * *} \mathrm{P}<0.001$. (C) Representative photomicrographs showing TUNEL-positive cells at $\mathrm{x} 20$ or $\mathrm{x} 40$ magnification in experimental groups (red arrow). (D) After cells were treated with 5 or $10 \mu \mathrm{M}$ of OP-B for $24 \mathrm{~h}$, expression levels of caspase-3, Bax, Bcl-2 and $\beta$-actin were detected by western blot analysis. Images or data are shown from three independent experiments. 
A

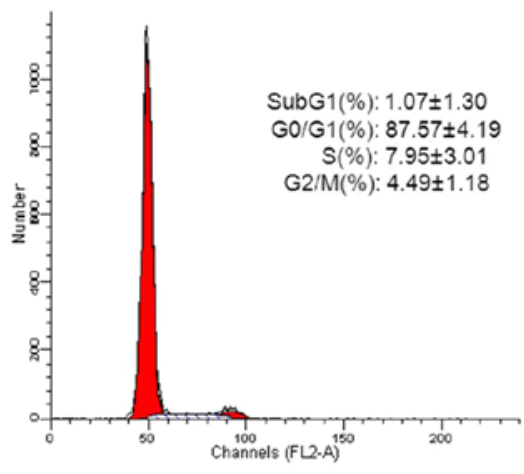

$0 \mu \mathrm{mol} / \mathrm{l}$

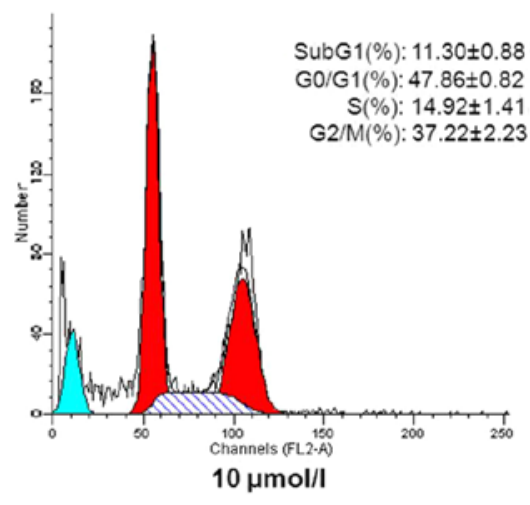

B

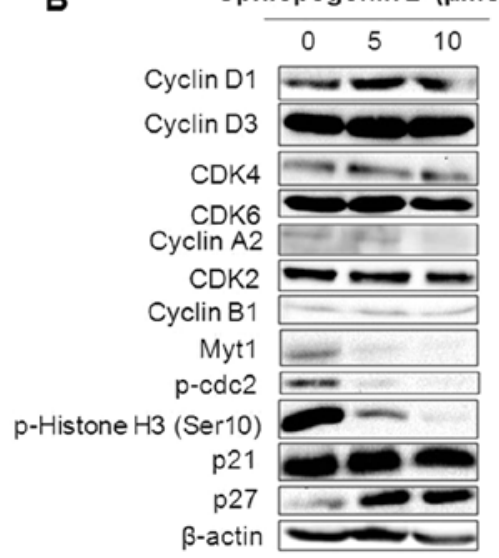

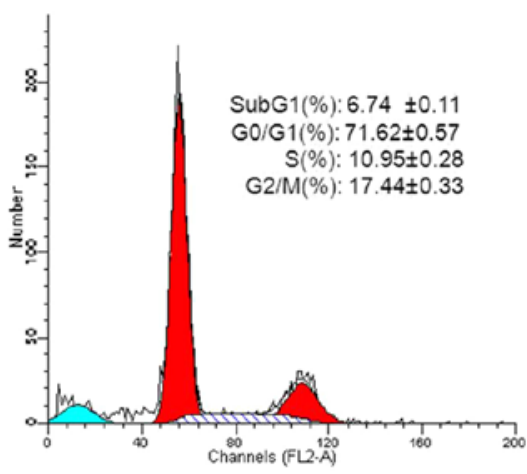

$5 \mu \mathrm{mol} / \mathrm{I}$

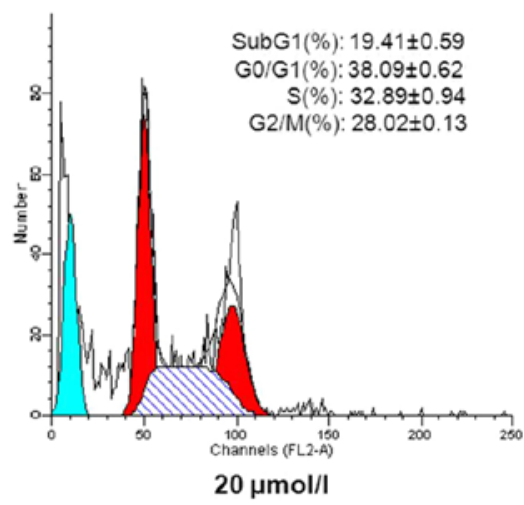

Ophiopogonin B $(\mu \mathrm{mol} / \mathrm{l})$

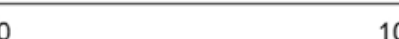

Figure 2. OP-B induces cell cycle arrest in S and G2/M phase and mitotic catastrophe in A549 cells. (A) A549 cells were treated with 5-20 $\mu \mathrm{M}$ of OP-B for $24 \mathrm{~h}$, then fixed in ethanol and stained with PI. The DNA content and percentage of cells in each cell cycle phase was determined by flow cytometry. Data are presented as the mean \pm standard error. (B) A549 cells were treated with or without $10 \mu \mathrm{M}$ of OP-B for $24 \mathrm{~h}$, and then expression levels of cyclin D1, cyclin D3, CDK4, CDK6, cyclin A2, CDK2, cyclin B1, Myt1, p-cdc2, p-Histone H3, p21 and p27 were detected by western blotting, $\beta$-actin was used as a loading control. (C) A549 cells treated with or without $10 \mu \mathrm{M}$ of OP-B for $24 \mathrm{~h}$ were then stained by DAPI and the nucleus morphology was observed by HCS (200) (multiploid of nucleus are pointed out with red arrows). The experiment was repeated three times and yielded similar results.

that OP-B-mediated autophagy promotes cell death in A549 cells.

Importantly, genetic inhibition of OP-B-mediated autophagy by transfection with Beclin1 siRNA also significantly decreased the cell distribution in Sub G1 phase exerted by OP-B in A549 cells (Fig. 3D). Together, the data indicated that OP-B-induced autophagy was the vital reason which resulted in apoptosis in A549 cells.

OP-B inhibits NSCLC xenograft growth in association with induced autophagy and apoptosis. To further authenticate the effects of OP-B described above, animal experiments were designed to test whether OP-B could induce autophagic and apoptotic cell death in vivo, and NSCLC xenografts in nude mice were established using A549 cells. The results showed that the growth of NSCLC tumors was significantly inhibited by treatment with OP-B at $75 \mathrm{mg} / \mathrm{kg}$ compared with the vehicle controls (Fig. 4A and B). Results of western blot assay showed that the high dose of OP-B significantly inhibited the phosphorylation of Histone H3 (Ser10), and at both of the doses of OP-B the levels of survivin and XIAP were inhibited (Fig. 4C). The tumors from OP-B-treated mice exhibited significantly increased expression of LC3; moreover, treatment with OP-B significantly induced cellular apoptosis in tumor tissues as evidenced by the TUNEL assay (Fig. 4D). These results indicated that OP-B could induce autophagic and 


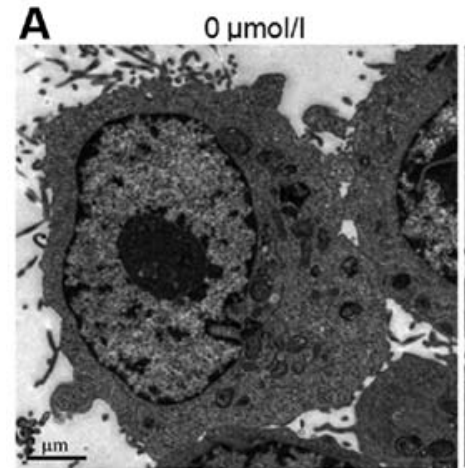

B

Ophiopogonin B $(10 \mu \mathrm{mol} / \mathrm{l})$

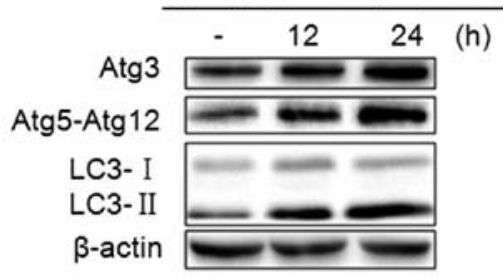

D
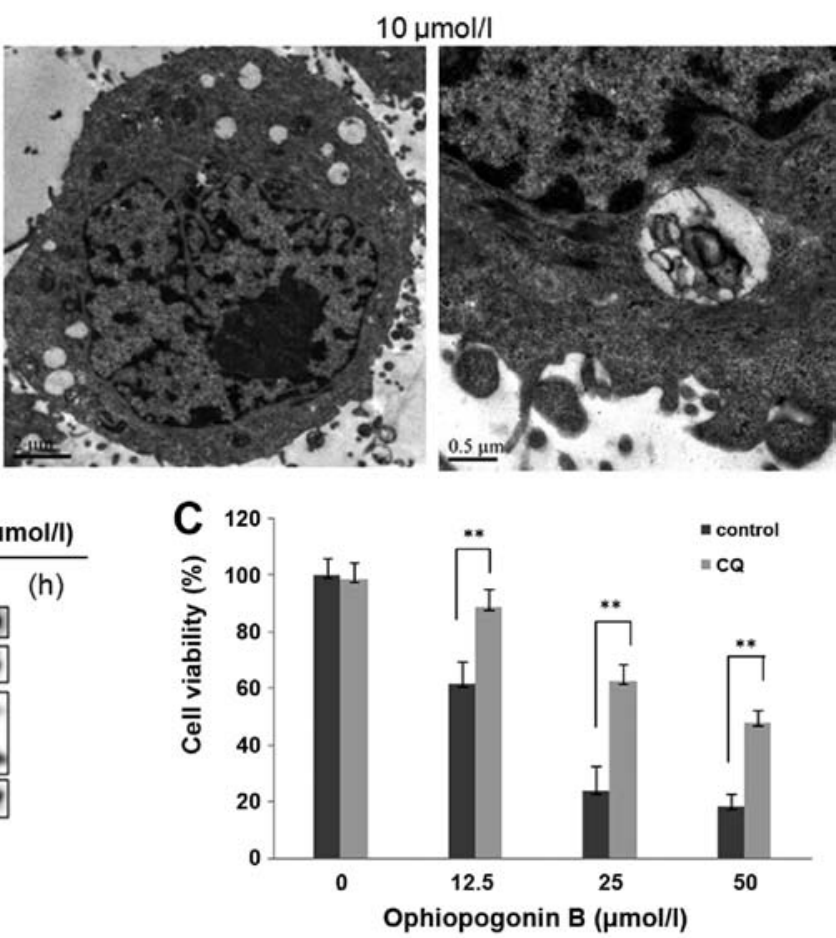
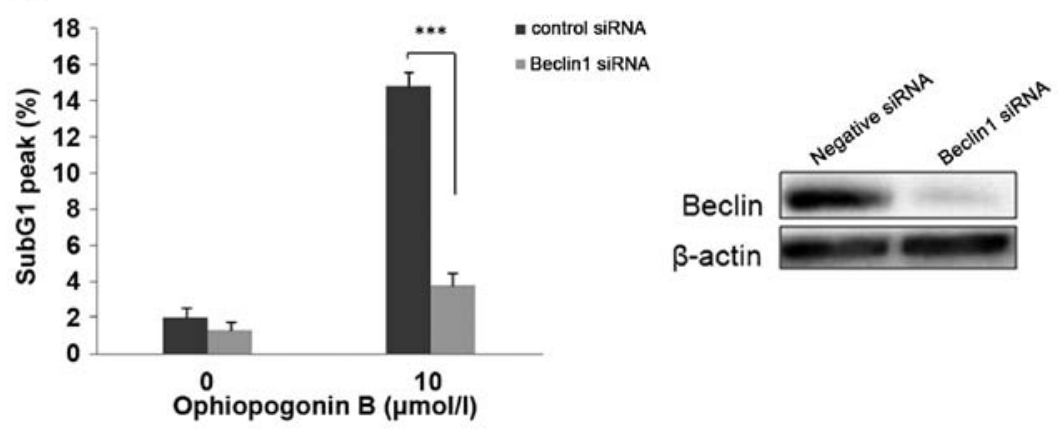

Figure 3. OP-B induces autophagy in A549 cells. (A) TEM examination of A549 cells treated with $10 \mu \mathrm{M}$ of OP-B for 24 h. (B) A549 cells treated with 5 and $10 \mu \mathrm{M}$ of OP-B for 12 or $24 \mathrm{~h}$ were analyzed by immunoblotting with antibodies against Atg3, Atg5-Atg12 complex, LC3 and $\beta$-actin. The experiment was repeated three times and yielded similar results. (C) After treatment with indicated concentrations of OP-B with or without CQ for 24 h, cell viability was measured by Trypan blue dye exclusion assay. (D) After transfection with negative siRNA or Beclin 1 siRNA for 36 h followed by treatment with $10 \mu \mathrm{M}$ OP-B for $12 \mathrm{~h}$, the percent of cells in sub G1 was analyzed by PI staining and flow cytometry. Error bars, $\mathrm{SD}$. ${ }^{*} \mathrm{P}<0.05 ;{ }^{* *} \mathrm{P}<0.01 ;{ }^{* * *} \mathrm{P}<0.001 . \mathrm{Data}$ are means of triplicate experiments.

apoptotic cell death in A549 cells in vivo, which may be an important mechanism underlying the antitumor activities of OP-B.

\section{Discussion}

In our previous study, we reported that OP-B inhibited proliferation of a panel of NSCLC cell lines; importantly, it showed a relatively low $\mathrm{IC}_{50}$ in $\mathrm{A} 549$ cells $(2.63 \mu \mathrm{M})(7)$. As approximately $40 \%$ of lung cancers are adenocarcinomas in clinic, in the present study we focused on the effect of OP-B on A549 cells in vitro and in vivo to further elaborate the underlying mechanisms of OP-B in NSCLC.

Firstly, positive labeling of Annexin V/PI and TUNEL staining both demonstrated that OP-B caused apoptosis in A549 cells (Fig. 1A-C), while detection of caspase-3, Bcl-2 and Bax showed that the apoptosis was caspase and mitochondrial independent (Fig. 1D).
Cell cycle arrest is another key factor preventing tumor growth. In the previous study, we reported that OP-B induced cell cycle arrest in the G0/G1 phase in H157 and H460 cell lines (7). However, our current flow cytometric analysis in A549 cells showed that OP-B markedly suppressed the cell mitotic progression through arresting cells in the $S$ and G2/M phase; whereas, it dose-dependently induced the increase of cells in the Sub-G1 phase (Fig. 2A). It is well known that the eukaryotic cell cycle is regulated by the coordinated activity of CDK kinase complexes which form and are activated at specific cell cycle stage (17). These cyclin-CDK complexes often bind to the endogenous inhibitor $\mathrm{p} 21^{\mathrm{WAF} 1 / \mathrm{CIP} 1}$ or $\mathrm{p} 27^{\mathrm{KIP} 1}$, which inhibits their kinase activities and prevent cell cycle progression. Detection of these proteins showed that OP-B upregulated p27 ${ }^{\mathrm{KIP} 1}$, while downregulated Myt1, p-cdc2 and p-Histone H3 (Ser10) in A549 cells (Fig. 2B). Phosphorylation at Ser10, Ser28 and Thr11 of Histone H3 is tightly correlated with chromosome condensation during both mitosis and 
A Ophiopogonin B (mg/kg)
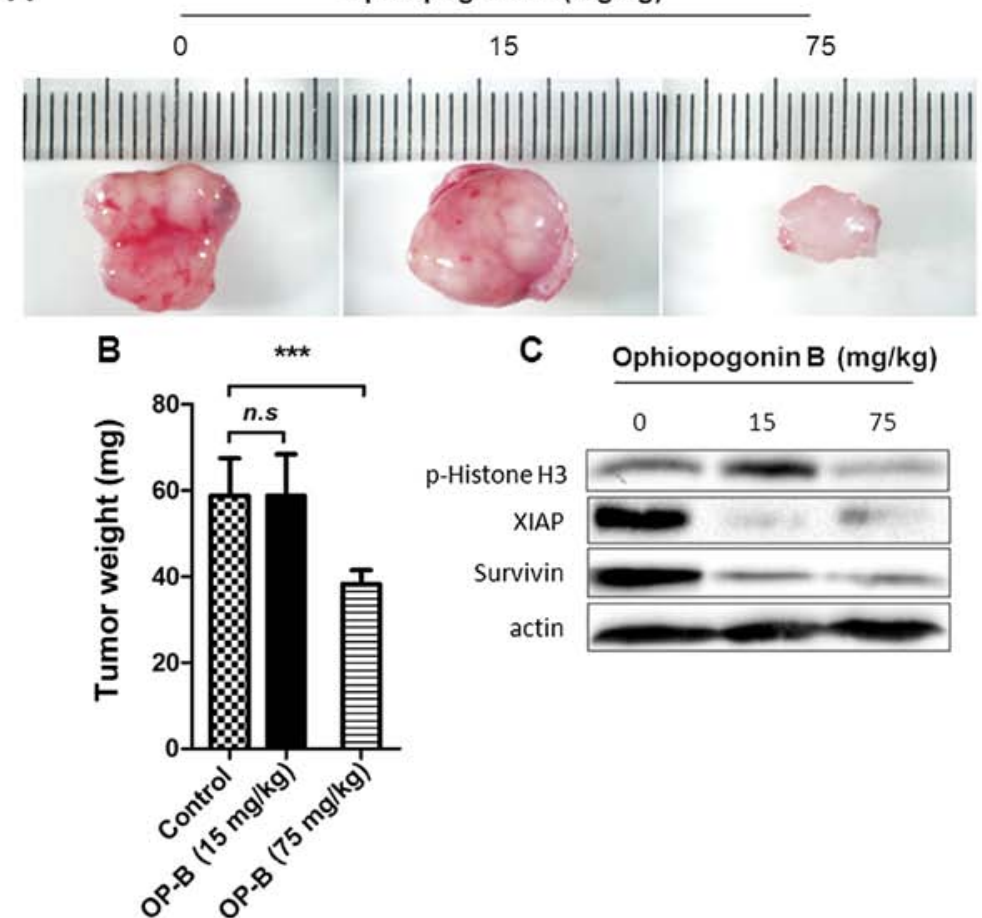

C Ophiopogonin B $(\mathrm{mg} / \mathrm{kg})$

D Ophiopogonin B (mg/kg)
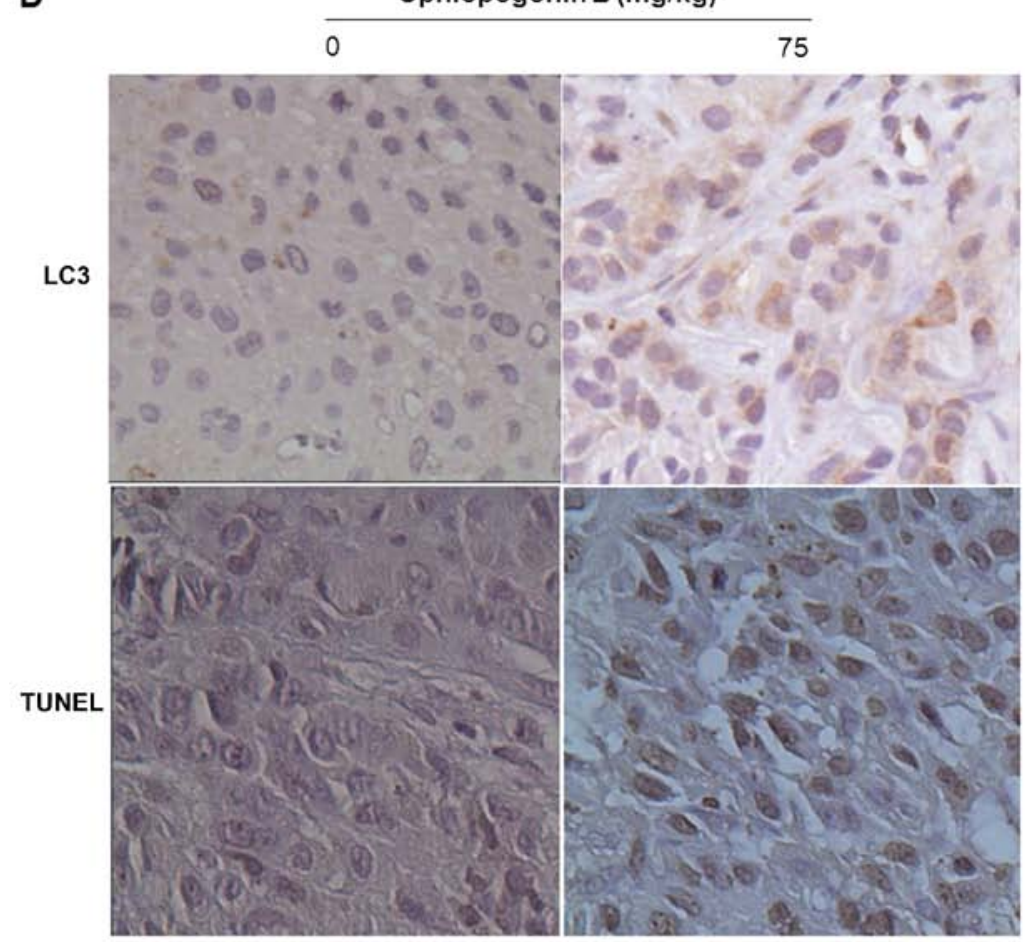

Figure 4. OP-B induces autophagy and apoptotic cell death in vivo. (A and B) Tumor size or tumor weight of vehicle-treated mice or $15 \mathrm{or} 75 \mathrm{mg} / \mathrm{kg}$ OP-B treated mice. (C) Western blot detection of p-Histone H3 (Ser10), survivin and XIAP in tumor cells treated with or without OP-B. (D) Immunohistochemical analysis of LC3 and TUNEL staining in both control and OP-B-treated A549 tumor tissues. Error bars, SD. ${ }^{*} \mathrm{P}<0.05 ;{ }^{* * *} \mathrm{P}<0.01 ;{ }^{* * * *} \mathrm{P}<0.001$.

meiosis (18), and singular phosphorylation of Histone $\mathrm{H} 3$ (Ser10) can act as part of a molecular mechanism driving mitotic chromosomal condensation during $\mathrm{M}$ phase entry (14). Further observation of the nucleus morphology by DAPI staining showed that OP-B treatment resulted in micronuclei formation in the cells, being the typical characteristic of mitotic catastrophe (Fig. 2C). Taken together, upregulation of p2 $7^{\mathrm{KIP} 1}$ and suppression of p-Histone $\mathrm{H} 3$ (Ser10) resulted in cell cycle arrest in S and G2/M phase, and further induced mitotic catastrophe in A549 cells.

Autophagy has a complex relationship with apoptosis, especially in tumor cell lines (19-22). Here, cell micro- 


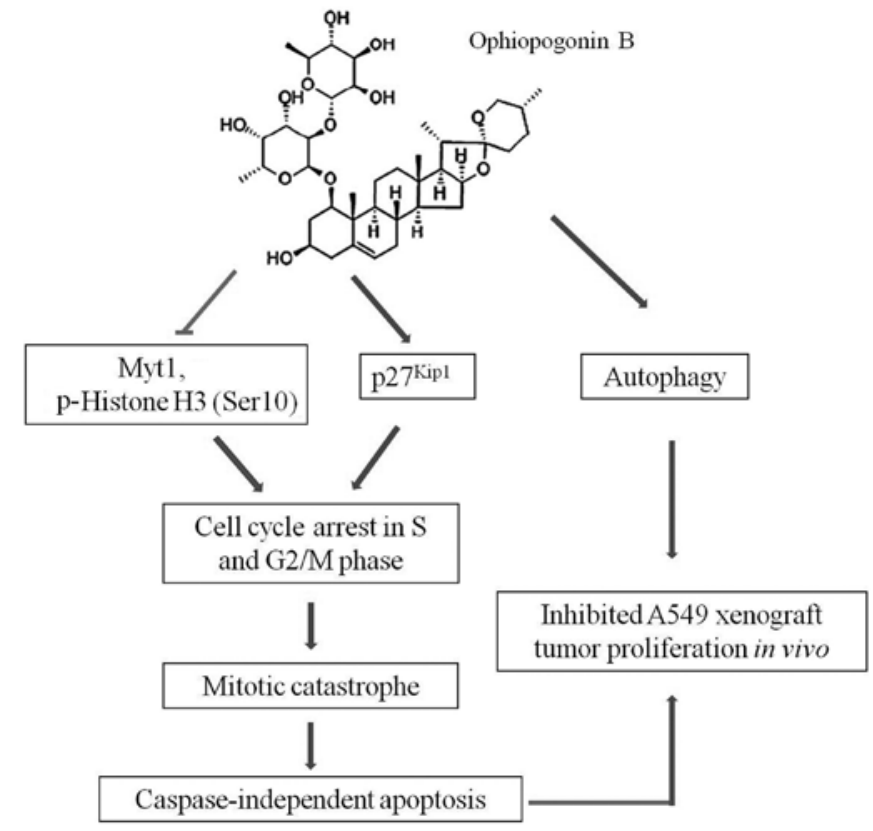

Figure 5. The proposed mechanism of ophiopogoninB (OP-B) in A549 cells. Inhibition of Myt1 and p-Histone H3 (Ser10) and upregulation of p27 ${ }^{\text {Kipl }}$ induced cell cycle arrest in S and G2/M phase, which further resulted in mitotic catastrophe, combined with induction of autophagy, it resulted in caspase-independent apoptosis in A549 cells in vitro and in vivo.

morphology observed by TEM combined with detection of autophagy-related proteins by western blotting showed that OP-B also induced autophagy in A549 cells (Fig. 3A and B). Moreover, lysosome inhibitor CQ or Beclin1 siRNA knockdown were shown to both attenuate the level of cell apoptosis (Fig. 3C and D), indicating that autophagy promoted apoptosis in A549 cells. As known, not only caspases, other proteases can also execute programmed cell death, and they can be directed by several cellular organelles, including mitochondria, lysosomes, and the ER, which can collaborate with each other or act independently. Different death routes may overlap and several characteristics may be displayed at the same time (6,23-25).

For these multiple action of OP-B on A549 cells in vitro, we used 15 and $75 \mathrm{mg} / \mathrm{kg}$ of OP-B in A549 xenografts nude mice to detect its effect in vivo. The results showed that OP-B exerted significant inhibition of tumor proliferation (Fig. 4A and $\mathrm{B}$ ). The mechanism may be related with phosphorylation inhibition of Histone H3 (Ser10) and suppression on survivin and XIAP (Fig. 4C), which resulted in mitotic catastrophe and further induced autophagy and apoptosis (Fig. 4D) of the tumor cells.

Taken together, other than its action on H157 and H460 cells, OP-B exerted overlapped types of cell death in A549 cells, including mitotic catastrophe, autophagy and caspaseindependent apoptosis (Fig. 5). The difference may due to the unique characteristics of different cell lines. As having the distinguishing trait of type II pulmonary epithelial cells, A549 cells usually form confluent monolayers during growth, and they express P450 IA1 and P450 IIB6 isozymes, which facilitate delivery of macromolecules and are important for metabolism of drugs (26). This may be the reason for A549 cells to be more sensitive to OP-B. Thus, OP-B may be developed as an alternative agent that can be used for the classification and treatment of NSCLC.

\section{Acknowledgements}

The present study was supported by grants from the Priority Academic Program Development of Jiangsu Higher Education Institutions (PAPD), the Natural Science Foundation of Jiangsu Province (BK20131415 to M.C.), the National Natural Science Foundation of China (81503374 to M.C.), the Natural Science Foundation of Jiangsu Province (BK20151003 to Y.G.), China and Europe taking care of healthcare solutions, $\mathrm{CHETCH}$ Grant Agreement Number: PIRSES-GA-2013-612589 (to X.Z.).

\section{References}

1. Dresler CM, Fratelli C, Babb J, Everley L, Evans AA and Clapper ML: Gender differences in genetic susceptibility for lung cancer. Lung Cancer 30: 153-160, 2000.

2. Jacobson FL, Austin JHM, Field JK, Jett JR, Keshavjee S, MacMahon H, Mulshine JL, Munden RF, Salgia R, Strauss GM, et al: Development of The American Association for Thoracic Surgery guidelines for low-dose computed tomography scans to screen for lung cancer in North America: Recommendations of The American Association for Thoracic Surgery Task Force for Lung Cancer Screening and Surveillance. J Thorac Cardiovasc Surg 144: 25-32, 2012

3. Siegel R, Naishadham D and Jemal A: Cancer statistics, 2013. CA Cancer J Clin 63: 11-30, 2013.

4. Xiong F, Jiang M, Huang Z, Chen M, Chen K, Zhou J, Yin L, Tang Y, Wang M, Ye L, et al: A novel herbal formula induces cell cycle arrest and apoptosis in association with suppressing the PI3K/AKT pathway in human lung cancer A549 cells. Integr Cancer Ther 13: 152-160, 2014.

5. Zhu J, Chen M, Chen N, Ma A, Zhu C, Zhao R, Jiang M, Zhou J, Ye L, Fu H, et al: Glycyrrhetinic acid induces G1-phase cell cycle arrest in human non-small cell lung cancer cells through endoplasmic reticulum stress pathway. Int J Oncol 46: 981-988, 2015.

6. Zhao R, Chen M, Jiang Z, Zhao F, Xi B, Zhang X, Fu H and Zhou K: Platycodin-D induced autophagy in non-small cell lung cancer cells via PI3K/Akt/mTOR and MAPK signaling pathways. J Cancer 6: 623-631, 2015.

7. Chen M, Du Y, Qui M, Wang M, Chen K, Huang Z, Jiang M, Xiong F, Chen J, Zhou J, et al: Ophiopogonin B-induced autophagy in non-small cell lung cancer cells via inhibition of the PI3K/Akt signaling pathway. Oncol Rep 29: 430-436, 2013.

8. Bröker LE, Kruyt FA and Giaccone G: Cell death independent of caspases: A review. Clin Cancer Res 11: 3155-3162, 2005.

9. Zhang JJ, Zhu Y, Xie KL, Peng YP, Tao JQ, Tang J, Li Z, Xu ZK, Dai CC, Qian ZY, et al: Yin Yang-1 suppresses invasion and metastasis of pancreatic ductal adenocarcinoma by downregulating MMP10 in a MUC4/ErbB2/p38/MEF2C-dependent mechanism. Mol Cancer 13: 130, 2014.

10. Mosmann T: Rapid colorimetric assay for cellular growth and survival: Application to proliferation and cytotoxicity assays. J Immunol Methods 65: 55-63, 1983.

11. Sherr CJ: The Pezcoller lecture: Cancer cell cycles revisited. Cancer Res 60: 3689-3695, 2000.

12. Malumbres M and Barbacid M: To cycle or not to cycle: A critical decision in cancer. Nat Rev Cancer 1: 222-231, 2001.

13. Lew DJ and Kornbluth S: Regulatory roles of cyclin dependent kinase phosphorylation in cell cycle control. Curr Opin Cell Biol 8: 795-804, 1996.

14. Hendzel MJ, Wei Y, Mancini MA, Van Hooser A, Ranalli T, Brinkley BR, Bazett-Jones DP and Allis CD: Mitosis-specific phosphorylation of histone $\mathrm{H} 3$ initiates primarily within pericentromeric heterochromatin during G2 and spreads in an ordered fashion coincident with mitotic chromosome condensation. Chromosoma 106: 348-360, 1997.

15. Mizushima N, Yoshimori T and Ohsumi Y: The role of Atg proteins in autophagosome formation. Annu Rev Cell Dev Biol 27: 107-132, 2011. 
16. Iwai-Kanai E, Yuan H, Huang C, Sayen MR, Perry-Garza CN, Kim L and Gottlieb RA: A method to measure cardiac autophagic flux in vivo. Autophagy 4: 322-329, 2008.

17. Jin YH, Choi J, Shin S, Lee KY, Park JH and Lee SK: Panaxadiol selectively inhibits cyclin A-associated Cdk 2 activity by elevating p $21^{\mathrm{WAF} 1 / \mathrm{ClP1}}$ protein levels in mammalian cells. Carcinogenesis 24: 1767-1772, 2003.

18. Preuss U, Landsberg G and Scheidtmann KH: Novel mitosisspecific phosphorylation of histone $\mathrm{H} 3$ at $\mathrm{Thr} 11$ mediated by Dlk/ZIP kinase. Nucleic Acids Res 31: 878-885, 2003.

19. Eisenberg-Lerner A, Bialik S, Simon H-U and Kimchi A: Life and death partners: Apoptosis, autophagy and the cross-talk between them. Cell Death Differ 16: 966-975, 2009.

20. Mizushima N, Levine B, Cuervo AM and Klionsky DJ Autophagy fights disease through cellular self-digestion. Nature 451: 1069-1075, 2008.

21. Sui X, Kong N, Ye L, Han W, Zhou J, Zhang Q, He C and Pan H: p38 and JNK MAPK pathways control the balance of apoptosis and autophagy in response to chemotherapeutic agents. Cancer Lett 344: 174-179, 2014.
22. Codogno P and Meijer AJ: Autophagy and signaling: Their role in cell survival and cell death. Cell Death Differ 12 (Suppl 2): 1509-1518, 2005

23. Chen TS, Wang XP, Sun L, Wang LX, Xing D and Mok M: Taxol induces caspase-independent cytoplasmic vacuolization and cell death through endoplasmic reticulum (ER) swelling in ASTC-a-1 cells. Cancer Lett 270: 164-172, 2008.

24. Bröker LE, Huisman C, Span SW, Rodriguez JA, Kruyt FA and Giaccone G: Cathepsin B mediates caspase-independent cell death induced by microtubule stabilizing agents in non-small cell lung cancer cells. Cancer Res 64: 27-30, 2004.

25. Huisman C, Ferreira CG, Bröker LE, Rodriguez JA, Smit EF, Postmus PE, Kruyt FA and Giaccone G: Paclitaxel triggers cell death primarily via caspase-independent routes in the non-small cell lung cancer cell line NCI-H460. Clin Cancer Res 8: 596-606, 2002.

26. Foster KA, Oster CG, Mayer MM, Avery ML and Audus KL: Characterization of the A549 cell line as a type II pulmonary epithelial cell model for drug metabolism. Exp Cell Res 243: 359-366, 1998 